\title{
The Role of the Monkey Dorsal Pontine Nuclei in Goal-Directed Eye and Hand Movements
}

\author{
Konstantin Tziridis, Peter W. Dicke, and Peter Thier \\ Department of Cognitive Neurology, Hertie Institute for Clinical Brain Research, University of Tübingen, 72076 Tübingen, Germany
}

Prevailing concepts on the control of goal-directed hand movements (HM) have focused on a network of cortical areas whose early parieto-occipital stages are thought to extract and integrate information on target and hand location, involving subsequent stages in frontal cortex forming and executing movement plans. The substantial experimental results supporting this "cortical network" concept for hand movements notwithstanding, the concept clearly needs refinement to account for the surprisingly mild HM disturbances resulting from disconnecting the parieto-occipital from the frontal stages of the network. Clinical observations have suggested the cerebropontocerebellar projection as an alternative pathway for the sensory guidance of HM. As a first step in assessing its role, we explored the pontine nuclei (PN) of rhesus monkeys, trained to make goal-directed hand and eye movements guided by spatial memory. We were indeed able to delineate a distinct cluster of neurons in the rostrodorsal PN, activated by the preparation and the execution of hand reaches, close to but distinct from the region in which saccade-related neurons may be observed. The movement-related activity of HM-related neurons starts earlier than that of saccade-related neurons and both neuron types are usually effector specific, i.e., they respond only to the movement of the preferred effector. This is also the case when motor synergies involving both effectors are executed. Our findings support the notion of a distinct precerebellar, pontine visuomotor channel for hand reaches that is anatomically and functionally largely separated from the one serving eye movements.

\section{Introduction}

To successfully reach for an object of interest, information concerning its location and shape has to be extracted and integrated with information about the actor's hand. It is usually assumed that the extraction and integration of information needed for the programming of a successful hand movement (HM) relies on a network of cortical areas. Specifically, this concept assumes that early parieto-occipital stages extract information on target and hand location and serve subsequent stages in frontal cortex integrating sensory information with pertinent information about the actor's hand to form and execute coherent movement plans (Soechting and Flanders, 1989; Sakata and Taira, 1994; Caminiti et al., 1996; Murata et al., 2000; Battaglia-Mayer et al., 2001; Marconi et al., 2001; Rizzolatti and Luppino, 2001; Buneo et al., 2002). The solid experimental arguments supporting this "intracortical communication" concept for HM notwithstanding, the concept faces the problem that it cannot account for the relatively weak HM deficits that have been described to result from complete surgical disconnection of the frontal and parietal lobes of monkeys (Myers et al., 1962; Haaxma and Kuypers, 1975). As such disconnections should effectively interrupt intracortical transmission of visual information for the guidance of HM, we

\footnotetext{
Received Feb. 3, 2009; revised March 17, 2009; accepted April 16, 2009.

This work is supported by the Deutsche Forschungsgemeinschaft (SFB 550 A7). We thank N. Catz for his suggestions on this manuscript and U. Grosshennig for technical assistance.

Correspondence should be addressed to Peter Thier, Department of Cognitive Neurology, Hertie Institute for Clinical Brain Research, University of Tübingen, Hoppe-Seyler-Straße 3, 72076 Tübingen, Germany. E-mail: thier@uni-tuebingen.de.

DOI:10.1523/JNEUROSCI.0581-09.2009

Copyright $\odot 2009$ Society for Neuroscience $\quad$ 0270-6474/09/296154-13\$15.00/0
}

must assume that visual information may be able to reach the motor system by resorting to an alternative pathway involving subcortical components. Anatomic considerations suggest that this alternative might be offered by the pathways interconnecting cerebral cortex and the cerebellum. The cerebropontocerebellar projection is fed by any cerebrocortical area contributing to spatial vision and the guidance of movements (Glickstein et al., 1985, 1990). The cerebellum in turn projects back—among others- to motor and premotor cortex by way of specific parts of the thalamus (Hoover and Strick, 1999; Hoshi et al., 2005). Hence, visual information for the guidance of HM could reach motor cortex despite the intracortical disconnection. The notion that cerebropontocerebellar communication is important for the visual guidance of movement is supported by the fact that disease-related lesions at the level of the human basis pontis not only disturb goal-directed eye movements (Thier et al., 1991; Gaymard et al., 1993; Thier and Möck, 2006) but also cause HM deficits. These deficits are observed even in the absence of concomitant lesions of neighboring corticospinal projections whose disruption leads to disturbances of strength rather than the dysmetria and clumsiness of HM associated with lesions of the more mediodorsal and also ventrolateral nuclear parts of the basis pontis (Schmahmann et al., 2004b). Why do pontine nuclei (PN) lesions give rise to such deficits and what are the specific roles of cerebrocerebellar communication and intracortical communication in the visual guidance of hand movements? To come up with answers to these questions we explored the PN of rhesus monkeys trained to make isolated hand, eye, or eye plus hand movements to memorized locations. Here we describe the features of a newly discovered set of pontocerebellar 


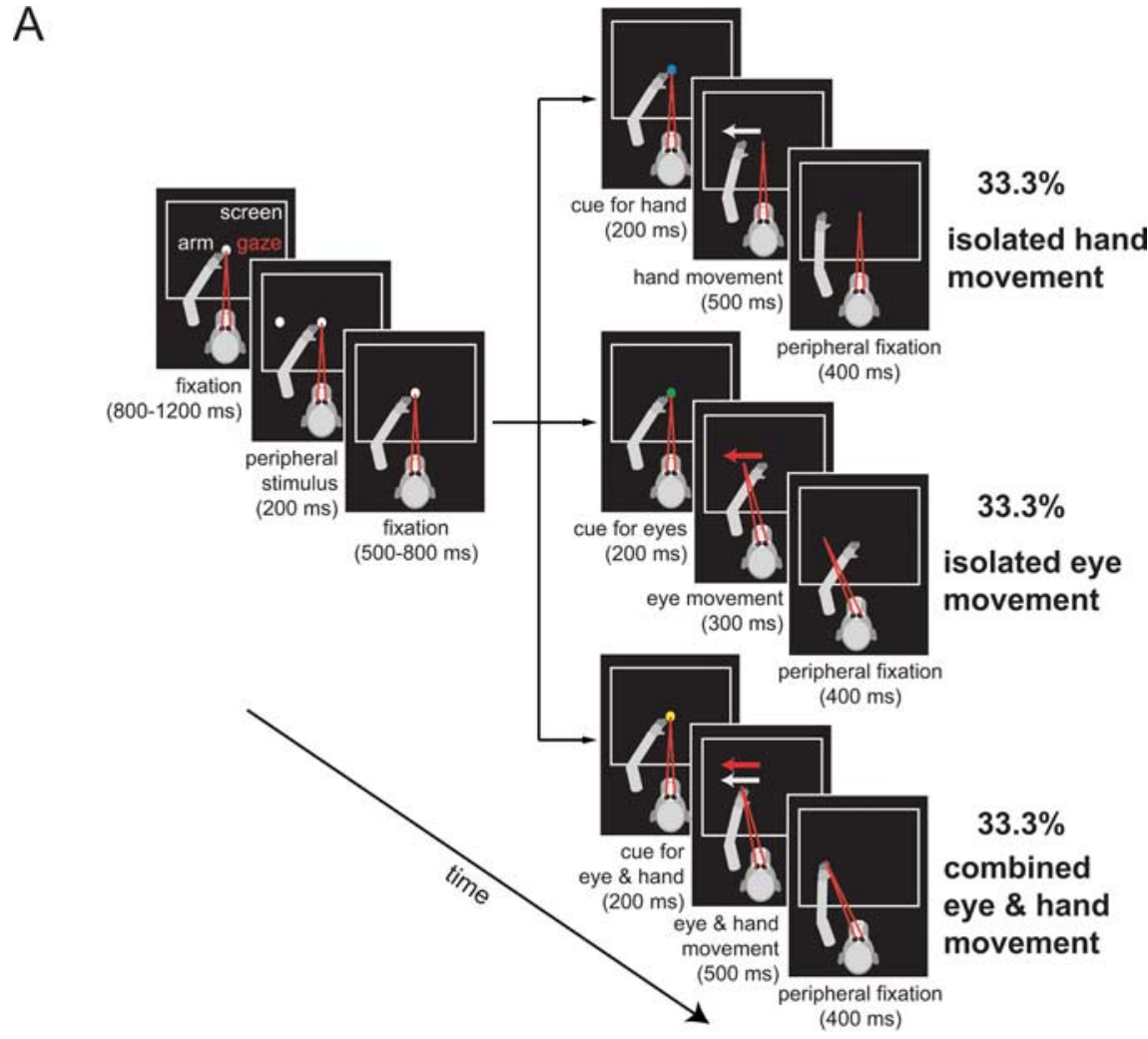

B
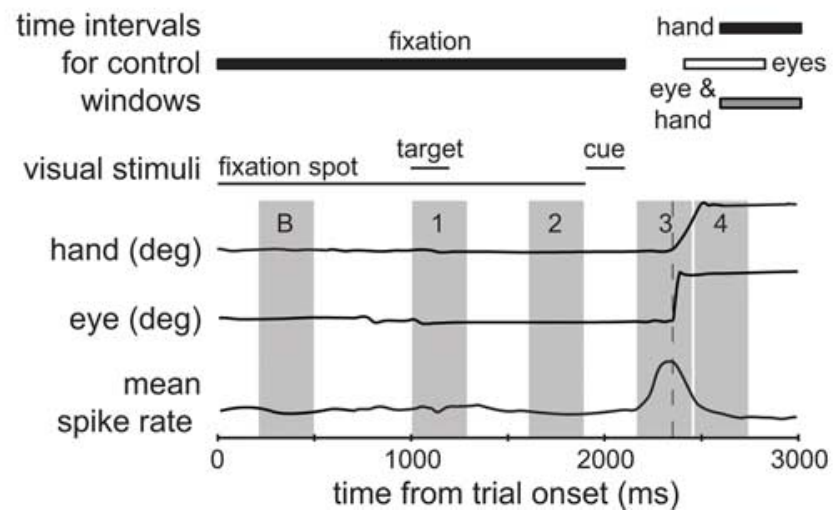

Figure 1. Paradigm and time intervals used for analysis. $A$, Sequence of events in the task. Each panel refers to a specific period of the monkeys' task; the durations of the respective segments are given in brackets below the panels. The arm is shown in gray, gaze direction in red, and the white rectangle symbolizes the computer CRT screen. After a variable time of fixation a white dot was presented in the periphery for $200 \mathrm{~ms}$ in one of eight possible directions. The monkey had to maintain fixation until the central fixation point changed color to blue to cue a hand movement, to green to cue a saccade, or to yellow to indicate a combined eye and hand movement. It was extinguished $200 \mathrm{~ms}$ later, the signal to start the requested movement. After reaching the target position with the correct effector the monkey had to maintain fixation of the peripheral location. After a successful trial the monkey was rewarded by a drop of liquid. $\boldsymbol{B}$, Definition of time intervals used for the quantification of neuronal responses. From top to bottom the rows show (1) the time intervals for the position control windows described in $A$, (2) the timing of the visual stimuli involved (fixation spot, peripheral target, and effector cue), (3) an exemplary trace of hand position (horizontal component only for hand movement to the right), (4) an exemplary trace of eye position (horizontal component only for eye movement to the right), and (5) an illustration of a typical response of a pontine neuron related to a movement. The gray areas delineate the various intervals used to quantify discharge rates in particular sequence of the task sequence, $B=$ baseline $(200-500 \mathrm{~ms}), 1=$ visual response $(1000-1300 \mathrm{~ms}), 2=$ preparatory response $(1600$ $1900 \mathrm{~ms}), 3=$ movement response $(2200-2500 \mathrm{~ms}), 4=$ position response $(2500-2800 \mathrm{~ms})$. deg, Degree.

neurons in the rostrodorsal PN, activated by the preparation and the execution of hand reaches.

\section{Materials and Methods}

Subjects and surgery. We recorded single units from two male rhesus monkeys (Macaca mulatta) of 8-10 kg (monkeys B and N). Both were implanted with an MRI-compatible titanium head-post and a titanium recording chamber (inner diameter of $28 \mathrm{~mm}$ ) centered on the sagittal midline above occipital cortex. The center of the chamber was at stereotactic coordinates $-28 \mathrm{~mm}$ from the interaural line. It had an inclination of $40^{\circ}$ in the sagittal plane toward the caudal pole, aiming at the dorsal pontine nuclei (DPN). The correct position of the chambers was verified by postimplantation MRI scans. The monkeys were also equipped with scleral "search coils" for the measurement of eye movements as described previously (Judge et al., 1980). Surgery was performed under combination anesthesia with isoflurane and remifentanil and monitoring of all relevant vital parameters (body temperature, $\mathrm{CO}_{2}$, blood oxygen saturation, blood pressure, ECG). After surgery, monkeys were supplied with opioid analgesics (buprenorphine) until they had fully recovered. One of the two monkeys was perfused intracardially with $4 \%$ paraformaldehyde under deep barbiturate anesthesia at the end of the electrophysiological studies to retrieve the recording sites in the pontine nuclei (see below). All animal preparations and procedures fully complied with the National Institutes of Health Guide for Care and Use of Laboratory Animals, and were approved by the local committee overseeing animal experiments.

Setup, timing, and spatial configuration of the stimuli. Eye movements of the monkeys were recorded by sampling the search-coils signals at $1 \mathrm{kHz}$ (spatial resolution $<0.1^{\circ}$ visual angle). Hand movements were tracked in $3 \mathrm{D}$ by triangulating the position of an ultrasound emitter attached to the monkey's hand deploying the Zebris CMS10 ultrasonic tracking system (Zebris Medical). The system accommodated a temporal resolution of $10 \mathrm{~ms}$ and a spatial resolution $<1 \mathrm{~mm}$.

A CRT monitor (CM20MKR, Tatung, 20 inch diagonal, $1024 \times 768$ resolution, $72 \mathrm{~Hz}$ refresh rate) was placed in front of the monkey in reaching distance $(33 \mathrm{~cm}$ nose-to-screen distance). The monkeys were trained to tolerate the restraining of one arm to the monkey chair as well as the placement of the Zebris ultrasound emitter onto the hand of the other arm. It was attached to a soft band yoking the three middle fingers at the level of the proximal interphalangeal joint such as to lie above the middle finger. Monkey B used his right hand for the movements while monkey $\mathrm{N}$ preferred his left hand. The emitter signal was associated with hand position by asking the monkey to move his hand to predefined locations on the screen cued by small dots of light (diameter $20 \mathrm{~min}$ of arc). A corresponding procedure was used to calibrate the search coil signal. During experiments, the eyes and the hand had to stay inside quadratic position windows whose size was typically $3 \times 3^{\circ}$ for the hand and $2 \times 2^{\circ}$ for the eyes if the desired location was central and $5^{\circ}$ and $4^{\circ}$, respectively, if it was peripheral.

Monkeys learned to comply with three experimental paradigms: memory-guided saccades, memory-guided hand movements, and combined memory-guided eye and hand movements. To start a trial (Fig. $1 A$ ) the monkeys had to fixate a white dot (diameter $20 \mathrm{~min}$ of arc) located in the center of the black frontoparallel CRT screen and at the 
same time to touch the screen close to this position without occluding the dot. After a period varying from 800 to $1200 \mathrm{~ms}$, a second white dot serving as target (diameter 20 min of arc) was flashed for $200 \mathrm{~ms}$ in one of eight possible peripheral locations. In most of the experiments the eccentricity chosen was $10^{\circ}$ and only occasionally smaller or larger eccentricities selected from a range of $8^{\circ}$ to $15^{\circ}$ were used. The direction was chosen in a pseudorandomized order at intervals of $45^{\circ}$. Monkeys had to maintain fixation of the central fixation dot during the presentation of the peripheral cue and the subsequent "memory period" of 500-800 ms after the termination of the peripheral stimulus. Two hundred milliseconds before the end of the "memory period," the central dot changed color, the color change informing the monkey on the type of action required. A change to green indicated that an isolated memoryguided eye movement without any hand movement would be required; a change to blue color indicated an isolated memory-guided hand movement without any eye movement and a switch to yellow defined a combined eye and hand movement to the remembered location. The subsequent disappearance of the fixation dot served as the go signal for the monkeys to execute the requested movement. After this go signal, the monkeys had 300 or $500 \mathrm{~ms}$ to execute the required eye and/or hand movement. After having reached the eccentric target position either with the eyes and/or the hand, they had to keep fixation there for $400 \mathrm{~ms}$ before finally being rewarded by the delivery of a drop of juice or water, depending on their individual preferences. Monkeys were motivated to work for these liquid rewards as free access to fluid outside the experiments was restricted according to standard procedures (Laule et al., 2003; technical bulletin and guidelines of the German Primate Center on fluid restriction in the shaping of behavior of rhesus monkeys in neurophysiological experiments, F.-J. Kaup and S. Treue, April 16, 2007). After a successfully executed trial monkeys had $1 \mathrm{~s}$ to reach the fixation point to start the next trial. If he surpassed this limit, a time-out of $1 \mathrm{~s}$ followed. A trial was aborted, the data discarded and no reward delivered if the monkey violated one of the control windows or failed to use the effector(s) required. The three different classes of tasks were presented either in blocks or, alternatively, pseudorandomly intermingled. While in monkey $\mathrm{N}$ only intermingled trials were deployed, in monkey B trials organized in blocks were used in approximately the first third of the experiments, followed by intermingled trials in the remainder of the experiments. We did not find any statistically significant differences in reaction times and in movement durations in the three paradigms between the random design and the block design in this monkey (Friedman's rank ANOVA; $p>0.05$ ).

Analysis of behavioral data. Movement onset was defined by the time at which the acceleration of the effector exceeded a predefined value $\left(2000 \% \mathrm{~s}^{2}\right.$ for the eyes and $500 \% \mathrm{~s}^{2}$ for the hand) within a time window of 50-300 ms (eye movements) and 50-500 ms (hand movements) after cue disappearance. The termination of the movement was determined as the time at which the acceleration had fallen below this threshold. The median durations and latencies of eye and hand movements in the different directions were compared by Friedman's rank ANOVAs individually for each monkey. Median durations and latencies pooled across directions were compared between the two monkeys by Mann-Whitney
$U$ tests. All analyses were performed with commercial software (Matlab, MathWorks; StatSoft).

Electrophysiological recording and analysis. Structural MRI was used in both monkeys to facilitate the localization of the PN contralateral to the hand used during the experiments. During initial recording sessions the approach to the PN was guided by the identification of characteristic electrophysiological "landmarks" such as the superior colliculus and the oculomotor nuclei as well as by the identification of characteristic visual and eye movement-related (EMR) responses established by previous work on the dorsal pontine nuclei (Suzuki et al., 1990; Dicke et al., 2004; Thier and Möck, 2006). Extracellular action potentials were recorded with self-made glass-coated platinum-tungsten electrodes (diameter 80 $\mu \mathrm{m}$, impedance 1-2.5 M $\Omega$ ) using a 5-probe multielectrode system (Thomas Recording). Spikes of well isolated single units were classified online by a Multi Spike Detector (MSD, Alpha Omega Engineering) exploiting a template matching algorithm (Wörgötter et al., 1986). We recorded up to two isolated units per electrode simultaneously from up to five independently moved electrodes. The electrodes were arranged in a cross-like pattern with a distance of $150 \mu \mathrm{m}$ between neighboring electrodes. Only neurons recorded long enough for allowing the collection of at least eight trials per direction and condition were considered for analysis. The quantitative analysis of neuronal activity was based on 
perievent histograms aligned with respect to stimulus and to movement onset respectively. Neurons responding to saccadic eye movements but not to hand movements will be referred to as saccade related (SR), while neurons that responded to hand movements with or without responding to saccadic eye movements will be referred to as hand movement related (HMR).

As illustrated in Figure $1 B$ we compared the baseline activity (200-500 $\mathrm{ms}$ after trial onset) with the mean neural activity in four different time periods: (1) the time period of the presentation of the peripheral target (from peripheral stimulus onset to $100 \mathrm{~ms}$ after the end of the stimulus),

(2) the preparation time before the appearance of the colored cue (300 ms before cue onset to cue onset), (3) the period of movement execution (200 ms before movement onset to $100 \mathrm{~ms}$ after movement onset), and (4) the period following the movement (100-400 ms after movement onset). Comparisons were based on paired $t$ tests with Bonferroni corrections for multiple comparisons. Sensitivity to smooth pursuit eye movements was assessed using step-ramp and circular pursuit paradigms as described by Dicke et al. (2004). Based on these comparisons, we could distinguish several different types of stimulus and/or movement-related neural responses: first, movement-related neurons with additional visual responses, e.g., to the peripheral stimulus. Second, movement-related neurons with burst-like responses during the movement period for saccades and/or hand movements. Third, movement-related neurons having a pause in their response during movement execution, and fourth, movement-related neurons with position-related activity. A neuron was considered directionally selective if a one-way ANOVA of the dependence of the movement-related discharge rates in the eight different directions showed significant differences between directions $(p<0.05)$. The analysis of event-related bursts (time of burst onset, time of peak discharge, burst duration, and mean discharge rate) of individual bursting neurons was based on a Poisson spike train analysis applied to single trials (Hanes et al., 1995). For neurons that displayed a burst for movements of only one effector, we quantified the discharge rate for the other effector by using the time points and preferred direction revealed by the burst analysis of the effector prompting bursts. If the neuron exhibited responses to the movement of both we analyzed the bursts independently. We compared the results of the two movement modalities (eye and hand movements) by paired Student's $t$ tests; movement-related bursts were considered to be effector specific if these tests showed a significant difference. The analysis of responses to combined movements for neurons responding only to one effector was based on temporally aligning trials with respect to the onset of the movement of this effector. For neurons that responded to both effectors, we calculated both hand and eye movement aligned perievent time histograms (PETHs) and compared the responses with the ones evoked by the respective isolated movements.

To obtain a measure of normalized mean burst responses relative to baseline, we calculated a Michelson contrast:

$$
I_{\text {change }}=\frac{A_{\text {burst }}-A_{\text {base }}}{A_{\text {burst }}+A_{\text {base }}},
$$

where $A_{\text {burst }}$ is the mean activity during the burst and $A_{\text {base }}$ is the mean baseline activity in a period having the same duration as the burst, starting $200 \mathrm{~ms}$ after trial onset. This measure was calculated for eye, hand, and combined movements separately. For analyzing the population of bursting neurons during isolated and combined eye and hand movements, we used Bonferroni-corrected paired Student's $t$ tests for the parameters $I_{\text {change }}$, time of burst onset, time of burst peak, and burst duration.

Anatomical reconstruction of recording sites. After having finished all recordings in monkey $\mathrm{B}$, we performed electrolytic lesions $(10 \mu \mathrm{A}$, anodal unipolar current, applied manually five times for $1 \mathrm{~s}$ ) at five separate locations within the region which had been explored electrophysiologically. Seven days later monkey B was perfused with paraformaldehyde fixative under deep barbiturate anesthesia. The brain was taken out and the pontine brainstem was sectioned parasagittally $(60 \mu \mathrm{m}$ slice thickness). Sections were Nissl stained. The positions of the electrolytic lesions served as reference points for the reconstruction of recording sites fol-
A

\section{0 eye movement related neurons}

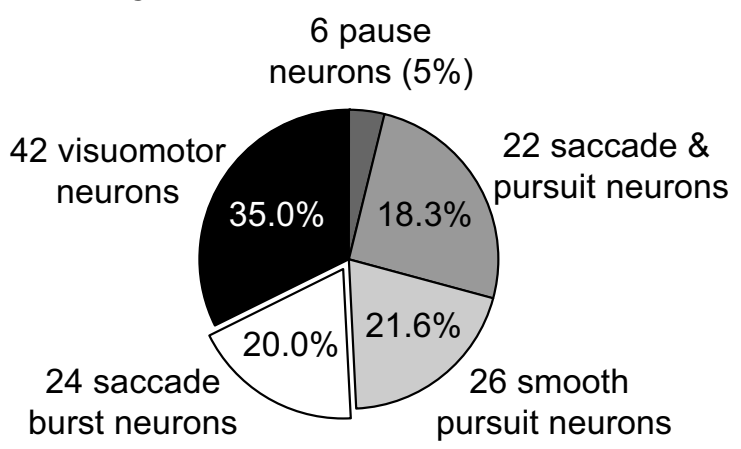

\section{B}

\section{2 hand movement related neurons}

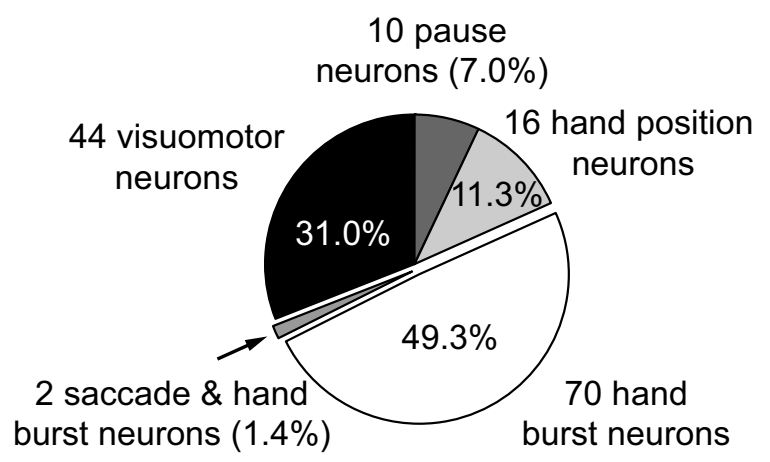

C

\section{preferred directions}

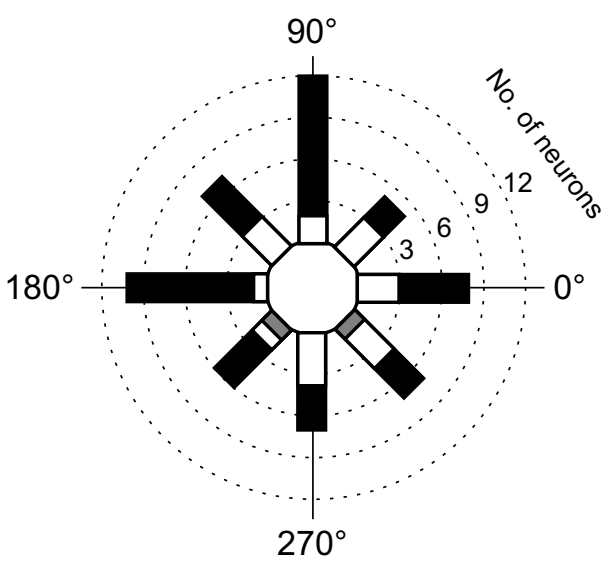

Figure 3. Numerical breakdown of all movement-related neurons. $A$, Eye movement-related units. $\boldsymbol{B}$, Hand movement-related units. In both $\boldsymbol{A}$ and $\boldsymbol{B}$, the percentage values give the relative fraction of the respective subgroup in relation to all eye and hand movement-related neurons, respectively. C, Polar histogram of the preferred directions of all 84 direction-selective burst neurons. The number of saccade-related burst neurons is represented by the white fraction of a bar, the number of hand movement-related neurons by the complementary black fraction. The gray fraction represents the two neurons that were activated by saccades as well as by hand movements.

lowing standard procedures, e.g., described by Dicke et al. (2004). The location of recording sites in monkey $\mathrm{N}$, which is still being used, were allocated to specific parts of the pontine brainstem by combining structural MRI, microdrive readings, properties of characteristic electrophys- 
iological landmarks and last but not least the lessons from the anatomy-physiology correlations obtained for monkey $\mathrm{B}$.

\section{Results}

Behavioral observations

The onset latencies and durations of saccades did not depend on direction (Friedman ANOVAs, $p>0.05$ ). On the other hand, the hand movement onset latencies as well as the hand movement durations showed a significant influence of direction (Friedman ANOVAs, $p<$ 0.001 ) for isolated hand movements as well as for those accompanied by eye movements. The specifics of the directional influence on hand movements depended on the monkey: the hand movement onset in monkey B was fastest and movement duration shortest for a direction of $45^{\circ}$ (latency for isolated hand movements ("isolated") $243.9 \mathrm{~ms}$, latency for combined hand movements ("combined") $235.2 \mathrm{~ms}$; duration isolated $110.0 \mathrm{~ms}$, duration combined 108.8 $\mathrm{ms})$, whereas monkey $\mathrm{N}$ preferred direction $180^{\circ}$ (latency isolated $240.2 \mathrm{~ms}$, latency combined $227.5 \mathrm{~ms}$; duration isolated $131.3 \mathrm{~ms}$, duration combined 133.6 $\mathrm{ms})$. These differences most probably reflect different hand preferences of the two monkeys: while monkey B used the right hand to reach to targets, monkey $\mathrm{N}$ preferred his left one, although the setup was symmetrical with respect to the sagittal midline of both monkeys. Exemplary hand and eye movement trajectories for movements in the eight directions in the frontoparallel plane of monkey B are depicted in Figure $2 \mathrm{~A}$.

Both monkeys exhibited significantly shorter hand movement onset latencies (Wilcoxon tests, $p<0.001$ for both monkeys) when hand movements were accompanied by eye movements compared with isolated hand movements (Fig. $2 B$ ), the same was true for the eye movement onset latencies (Wilcoxon tests, monkey B: $p<$ 0.01 , monkey $\mathrm{N}: p<0.05)$. Overall the onset latencies of monkey $\mathrm{N}$ were shorter than those of monkey B (Mann-Whitney $U$ tests, $p<0.001)$. On the other hand, the duration of hand movements with and without accompanying eye movements was longer in monkey N (Mann-Whitney $U$ tests, $p<0.001$ for both movement types) (Fig. 2C). In neither monkey did hand movement duration depend on the presence of accompanying eye movements (Wilcoxon tests, $p>$ 0.05 for both monkeys). Also, the duration of their eye movement remained unaffected by the absence or presence of hand movements (Wilcoxon tests, $p>0.05$ for both monkeys and both types of movement).

A position related HMR neuron

B

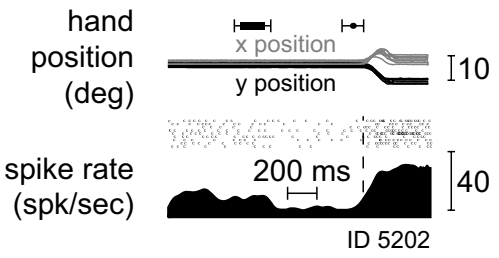

visual EMR neuron

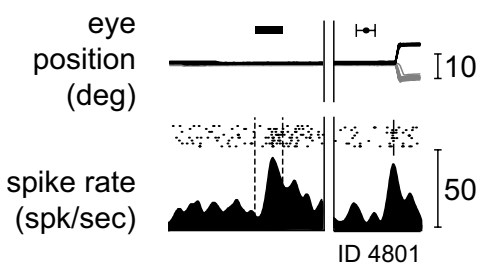

pursuit \& saccadic EMR neuron

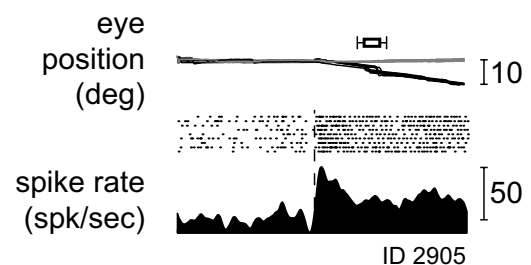

burst SR neuron

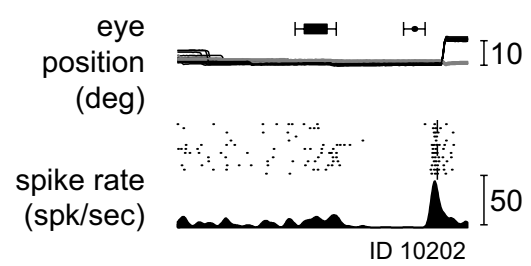

$\mathrm{H}$

Figure 4. Typical examples of responses of movement-related neurons in their respective preferred direction. Units are identified by their ID number. Time range of the occurrence of the peripheral stimulus (horizontal black bar with whiskers) and the time range of the go signal (black circle with whiskers) are depicted in the upper part of each subplot. $A$, HMR neuron responding to hand position. The top shows the $x$ - (gray) and $y$ - (black) components of the hand movement trajectories in the frontoparallel plane of the CRT aligned on movement onset. The bottom characterizes the firing of the neuron by raster plots as well as by filtered PETH (bin width $5 \mathrm{~ms}$, histogram second-order low-pass filtered at $100 \mathrm{~Hz}$ corner frequency) in spikes per second (spk/sec). The broken line indicates movement onset. $\boldsymbol{B}$, HMR neuron pausing before and during the hand movement. $\boldsymbol{C}$, EMR neuron responding not only to saccades but also to the flashed peripheral stimulus. The top shows the $x$ - (gray) and $y$ - (black) eye movement traces in degree over time. On the left of the solid vertical double line the traces and the neuronal responses are aligned on the onset of the peripheral stimulus (black bar; broken lines in histogram); on the right the movements and the responses are aligned on the saccade onset (broken vertical line in histogram). D, EMR neuron pausing before and during saccadic eye movements. Traces and neuronal responses are aligned on saccade onset. $\boldsymbol{E}$, EMR neuron responding for smooth pursuit and saccadic eye movements (latter not shown). The time interval of a $150 \mathrm{~ms}$ gap in the visual presentation of the moving pursuit target is indicated by an open bar with whiskers above the eye traces. $\boldsymbol{F}$, EMR neuron responding to smooth pursuit eye movements only (saccade trials not shown, but see the saccades to the initial fixation dot in the beginning of the trials). $G$, Neuron busting before and during saccadic eye movements (SR neuron) on which the responses are aligned (see supplemental Fig. S1, available at www. jneurosci.org as supplemental material). $\boldsymbol{H}, \mathrm{HMR}$ neuron busting before and during hand movements on which the responses are aligned. Only neurons that show the kind of bursts illustrated in $\mathbf{G}$ and $\boldsymbol{H}$ were used for further analyses (see supplemental Fig. S2, available at www.jneurosci.org as supplemental material). deg, Degree; pos., position.

Types of movement-related neurons in the dorsal pontine nuclei

We recorded 262 movement-related neurons (155 from monkey B, 107 from monkey N) from the dorsal pontine nuclei contralateral to the used hand which fell into two broad classes. The first class (Fig. 3A) comprised 120 neurons (45.8\%, 120/262) with eye 


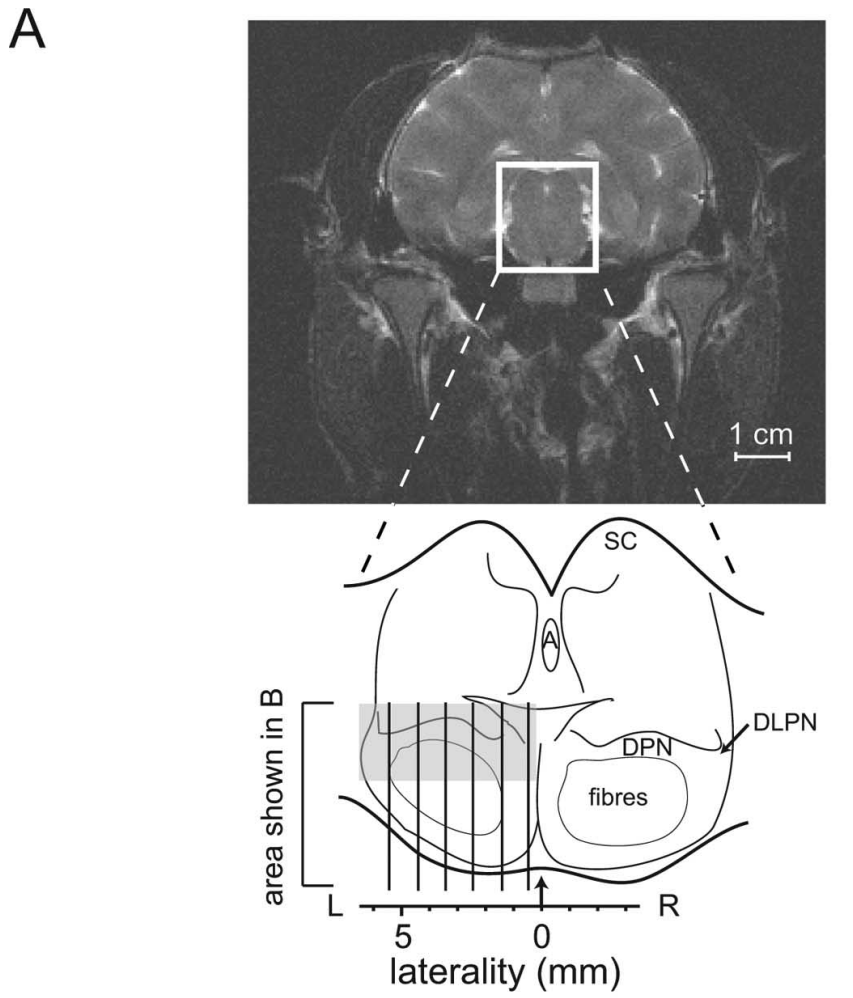

B

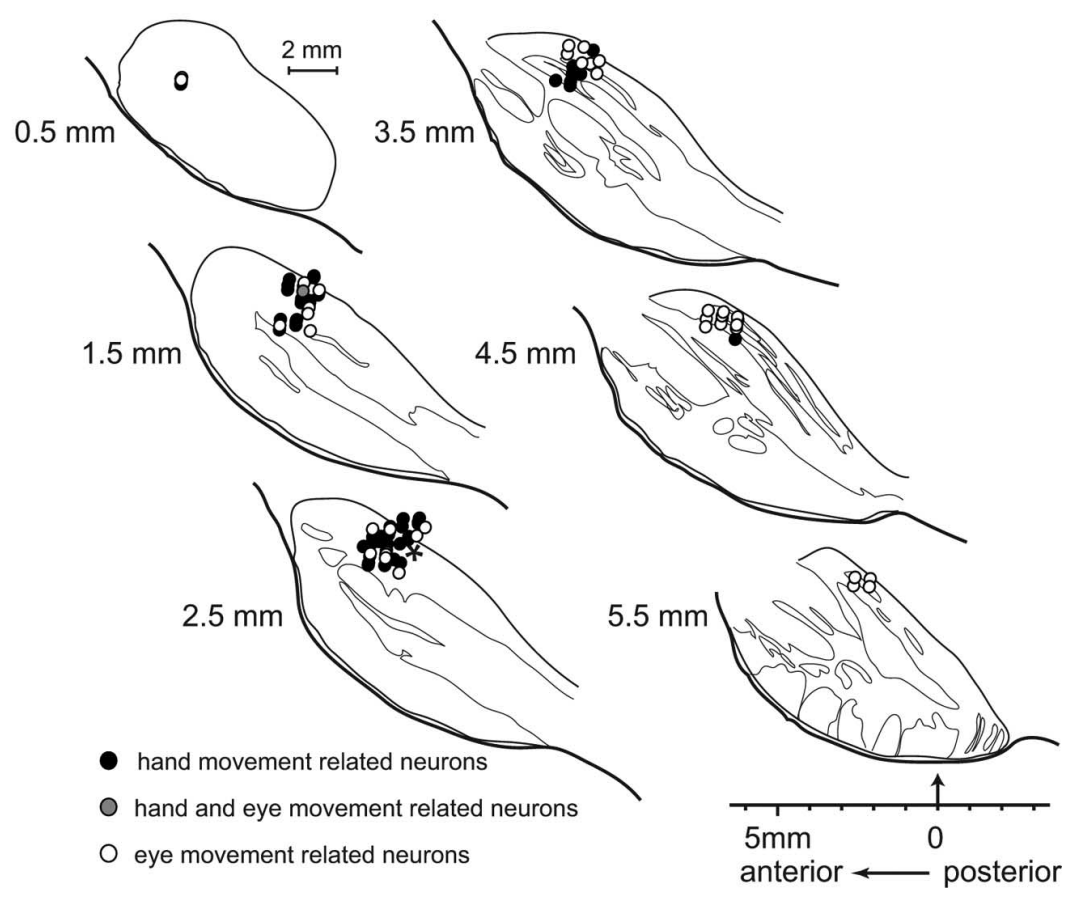

Figure 5. Structural MRI and reconstructed recording sites projected onto parasagittal histological sections for monkey B. $\boldsymbol{A}$, Structural MRI, frontal view centered on the middle of the recording chamber. The white rectangle indicates the location of the midbrain schema shown below. The vertical lines indicate the locations of the histological sections depicted in $\boldsymbol{B}$. The area explored electrophysiologically is delineated by a gray rectangle. A, Aqueductus cerebri; DLPN, dorsolateral pontine nuclei; SC, superior colliculus. $\boldsymbol{B}$, Reconstructed recording sites of movement-related units in the basilar pons of monkey $B$ plotted onto the outlines of the histological sections. The order of the drawings from top left to bottom right corresponds to sections taken from medial to lateral in $1 \mathrm{~mm}$ steps starting from $0.5 \mathrm{~mm}$ lateral from the midline (see $\boldsymbol{A}$ ); anterior to posterior on each section is shown from left to right in coordinates of the recording chamber. Thin lines demarcate the boundaries between pontine nuclei gray matter and fiber bundles. Black circles indicate the locations of hand movement-related neurons, open circles those of eye movement-related neurons, and gray circles the sites of neurons responding to both types of movements. Note that each symbol may actually represent more than one neuron with a particular preference for the type of movement. The asterisk indicates the location of one of the electrolytic lesions used to compute the positions of the recording sites. movement-related responses (EMR neurons). Within this class, 42 neurons (35\%, $42 / 120$ ) were also visually responsive to the presentation of the peripheral target. Twenty-four neurons (20\%, 24/120) within the class of EMR neurons responded with a burst before or during the saccade (SR burst neuron), 26 neurons $(21.6 \%, 26 / 120)$ were exclusively smooth pursuit related, and 22 neurons (18.3\%, $22 / 120$ ) were activated by both types of goal-directed eye movements. Finally, six neurons $(5 \%, 6 / 120)$ paused before or during the execution of saccades.

The second class (Fig. 3B) consisted of 142 HMR neurons $(54.2 \%, 142 / 262)$. They could be separated into six subclasses: 44 neurons $(31 \%, 44 / 142)$ also responded visually to the presentation of the peripheral target, 70 neurons $(49.3 \%, 70 / 142)$ responded with a burst before or during the time of the hand movement, but not before or during eye movements. Sixteen neurons $(11.3 \%, 16 / 142)$ were hand position dependent and 10 neurons (7\%, $10 / 142$ ) paused before or during the movement. We found only two hand movement-related bursting neurons $(1.4 \%, 2 / 142)$ which were also activated significantly before or during eye movements.

We also investigated the direction selectivity of the neuronal responses. As will be discussed in more detail later for the burst responses, visual as well as movement-related responses were typically direction selective. Twenty-two (91.6\%) of the 24 SR with exclusively saccade-related bursts, $62(88.5 \%)$ of the 70 HMR with exclusively hand movement-related bursts as well as the two neurons that showed bursts in both tasks exhibited a significant directional preference. The preferred directions of the 84 directionally selective SR and HMR with pure movement-related responses were uniformly distributed from 0 to $360^{\circ}$ (Fig. $3 C)$ [test according to Ajne (1968), $p>$ 0.05 for both classes]. In other words, pontine nuclei neurons from one side of the brainstem do not show a preference for movements to a particular side.

Figure 4 documents the diversity of neuronal response types found in the single pontine nuclei (PN) based on exemplary neurons, whose discharge patterns are represented by perievent time histograms (PETHs) and raster plots for their respective preferred direction. Figure $4 A$ shows the filtered PETH of an HMR neuron encoding hand position exhibiting a tonically elevated discharge after a hand movement of $270^{\circ}$. An HMR neuron, 
pausing just before movement initiation is shown in Figure $4 B$. Figure $4 C$ presents a saccade-related neuron that responded to the appearance of the peripheral target as well as firing a later saccade-related burst. Figure $4 D$ depicts an EMR neuron pausing at the time of the saccade and Figure $4 E$ illustrates an EMR neuron generating a burst-tonic response for constant velocity pursuit. This neuron also fired a saccaderelated burst (data not shown) in the memory-guided saccade task. Other EMR neurons were activated by smooth pursuit only and not by saccades (Fig. $4 F$ ). Finally, the last two neurons in this panel are examples of movement-related burst neurons, the one in Figure $4 G$ activated by saccades, the one in Figure $4 H$ by hand movements.

Figure 5 shows the reconstructed locations of the neurons in parasagittal sections of the brainstem of monkey $\mathrm{B}$. Movement-related neurons formed a cluster in the dorsomedial-dorsolateral pontine nuclei at the transition between the rostral most third of the pontine nuclei and the subsequent third of the pontine brainstem. Neurons responding to hand movements were in general located more medially than neurons responding to eye movements. Based on the circumstantial evidence available on the location of neurons in monkey $\mathrm{N}$, we assume that their topographical pattern fully corresponded to the one described for monkey B.

\section{The effector specificity of \\ movement-related bursts}

To quantify the movement-related bursts of hand movement-related neurons we focused on the largest group, the one comprising HMR neurons without concomitant visual responses. We did not consider bursts of visuomotor neurons as we wanted to avoid any remaining visual influence on our analysis of movement-related responses. The comparative analysis of saccade-related bursts was accordingly also restricted to those neurons without concomitant visual responses.

Figure $6 A$ depicts the burst profiles of a typical saccade-related burst neuron based on trials, which were aligned relative to saccade onset. This neuron discharged immediately before and during the saccade (black PETH, raster plot: black dots on white background), but did not respond to hand movements (gray PETH, raster plot: black dots on gray background). Its preferred saccade direction was downward $\left(270^{\circ}\right)$; the burst for this direction is demarcated in the figure by the two solid lines, giving the onset and the end of the
A
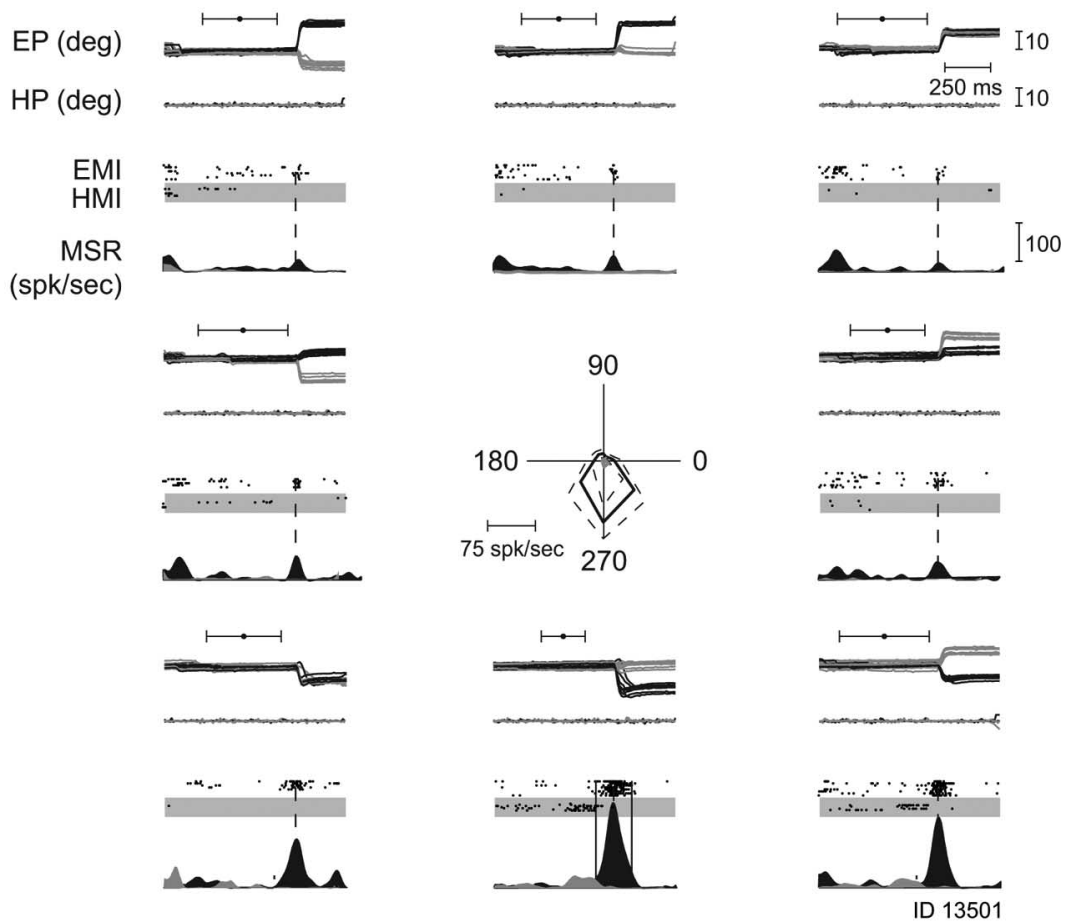

B
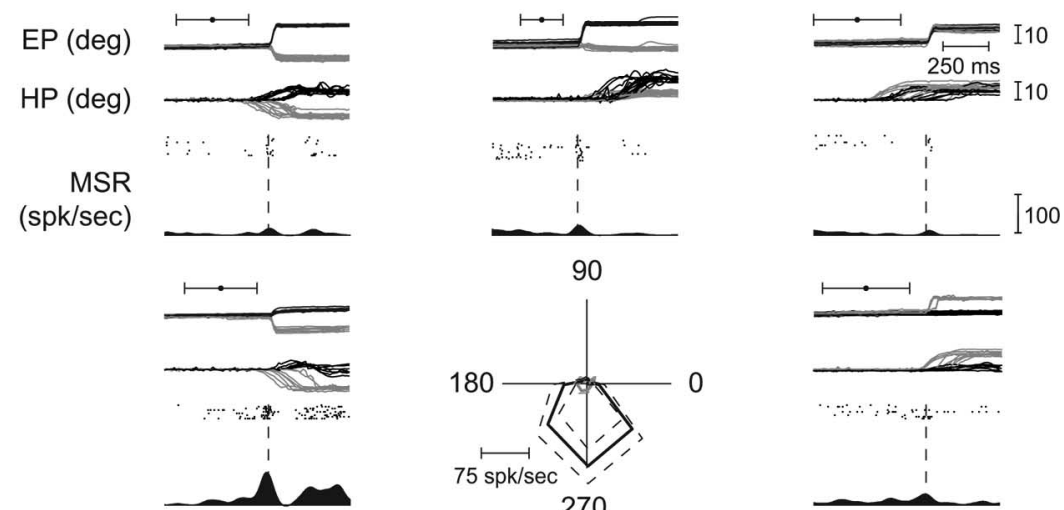

90
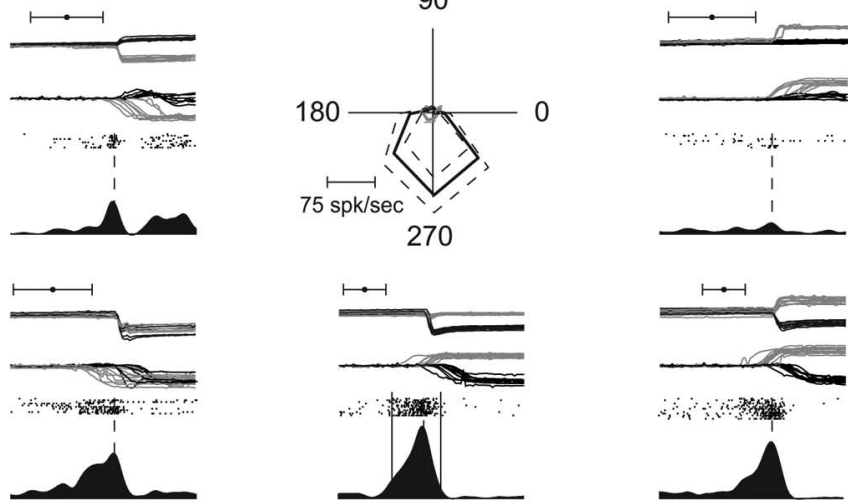

270
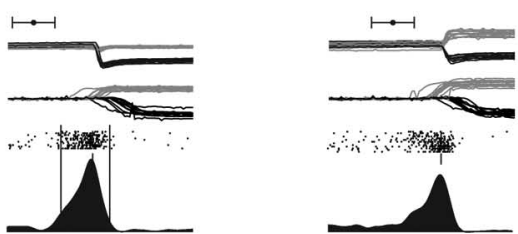

Figure 6. Exemplary eye movement-related neuron, preferring saccades. $\boldsymbol{A}$, Saccades in eight directions in the frontoparallel plane; for each direction the $x$ - (gray lines) and $y$-(black lines) positions of the eyes (EP, eye position) and the projections of the 3D hand trajectories onto the screen plane (HP, hand position) are shown. All traces are aligned on movement onset. The black dots with whiskers indicate the mean time \pm SD of the go signal. Response during isolated saccades (EMI, eye movements isolated) and hand movements (HMl, hand movements isolated) in eight directions in the frontoparallel plane. The neuronal discharge (MSR, mean spike rate) is represented by raster dots (saccades: black dots on white background, hand movements: black dots on gray background) and PETHs (saccades: black, hand movements: gray). Note: burst of activity during saccade trials, absence of discharge modulation during hand movement trials. Movement onset is indicated by a broken vertical line; onset and end of the bursts for the preferred direction of movement (here downward) are indicated by solid vertical lines. The polar plot in the center of the figure depicts the directional tuning curve of this neuron (rightward corresponds to $0^{\circ}$ ). The solid lines give the mean firing rates during saccades (black) and hand movements (gray) as function of direction for the time interval defined by burst onset and offset in the preferred direction. The broken black and gray lines give \pm SD of the discharge rate. $\boldsymbol{B}$, Responses of the same unit to yoked eye and hand movements, tested pseudorandomly interleaved with the movements shown in $\boldsymbol{A}$. For each direction the $x$ (gray lines) and $y$ - (black lines) positions of the eyes (EP) and the projections of the 3D hand trajectories onto the screen plane (HP) are shown. All traces are aligned on eye movement onset. The black dots with whiskers indicate the mean time \pm SD of the go signal. deg, Degree; spk/sec, spikes per second. 
A
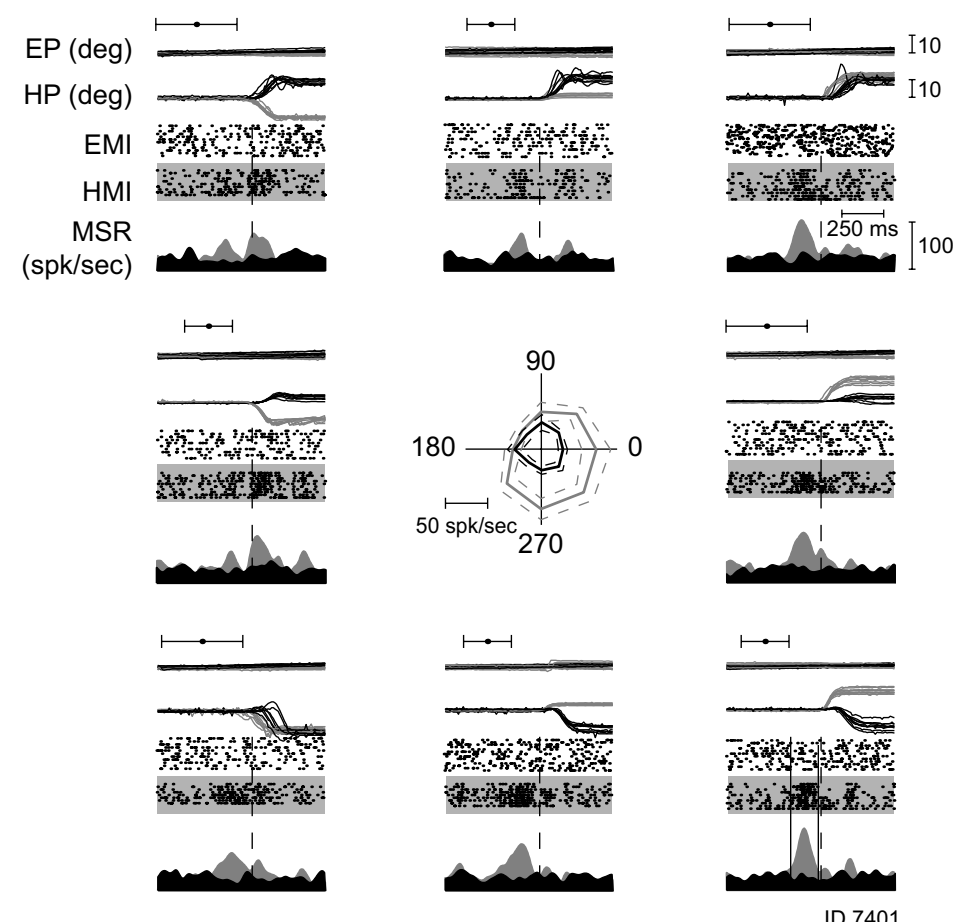

B
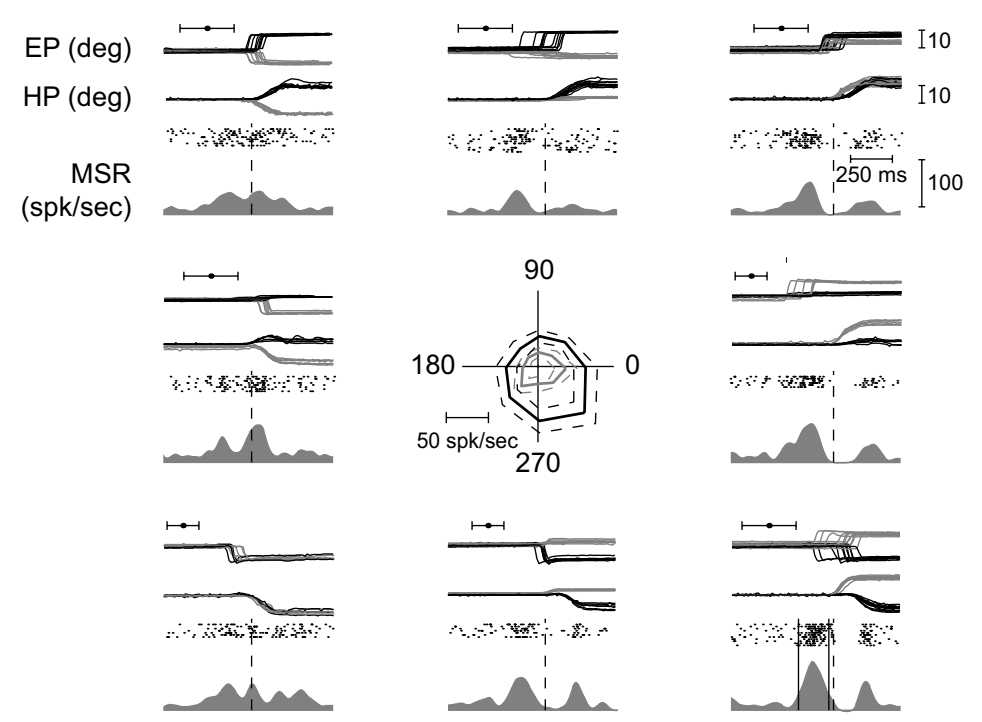

Figure 7. Typical hand movement-related neuron not responding to eye movements. $A$, Response during isolated saccades and hand movements in eight directions in the frontoparallel plane. Note: absence of discharge modulation during saccade trials, burst of activity during hand movement trials (for which the behavior is shown). Notations are as in Figure 6 A. $\boldsymbol{B}$, Responses of the same unit to yoked eye and hand movements, tested pseudorandomly interleaved with the movements shown in $A$. Notations are as in Figure $6 B$.

burst as determined by the Poisson analysis. The onset of the burst took place clearly before movement onset (indicated by the broken line) and ended $\sim 100 \mathrm{~ms}$ after the movement started.

Figure $7 \mathrm{~A}$ presents the example of an HMR burst neuron in the same format as the previous figure. This neuron preferred movements to the lower right $\left(315^{\circ}\right)$, however, of the hand and not the eyes (gray PETH, raster plot: black dots on gray background). Burst onset was $>200 \mathrm{~ms}$ before the hand movement onset and the end of burst occurred even before the beginning of the hand movement. As shown with the black PETH, the same neuron did not display any clear saccaderelated modulation, when the monkey was asked to make saccades, rather than hand movements. The results during combined eye and hand movements from Figures $6 B$ and $7 B$ will be described later.

Finally, Figure 8 shows one of the two neurons that were activated by saccades as well as by hand movements. During saccadic eye movements (Fig. 8A) this neuron exhibited comparatively weak saccade-related bursts before and during saccades with a directional preference for the lower right. The discharge pattern evoked by hand movements (Fig. 8B) looks very similar to the one for saccades.

The high degree of effector specificity of movement-related burst neurons is expressed by the plots of eye movementrelated discharge as function of hand movement-related discharge as shown in Figure $9 \mathrm{~A}$. It deploys the normalized response size measure $I_{\text {change }}$ with values of \pm 1 representing maximal burst signals relative to baseline (see Materials and Methods). Two clusters of neurons can be distinguished: SR neurons (the open circles) show strong responses during saccades and only weak responses during hand movements, whereas HMR neurons (filled rectangles) show the opposite behavior. The two neurons close to the bisectors, identified by the gray rectangles are the only ones we interpreted as being effector unspecific.

\section{Timing of movement-related bursts}

Figure $9 B$ shows the distributions of burst onset time (top) and burst peak time (bottom) with respect to movement onset for SR and HMR burst neurons. The measures of burst timing were based on the respective preferred direction. The median burst onset time of HMR burst neurons preceded movement onset by $93.5 \mathrm{~ms}$ and was significantly different from the median of SR burst neurons at $31.5 \mathrm{~ms}$ before movement onset (Mann-Whitney $U$ test, $p<$ $0.001)$. Both medians were significantly different from the time of movement onset (Wilcoxon signed rank test, $p<0.001$ for both). Correspondingly, the median burst peak time of HMR burst neurons preceded movement onset by $52.5 \mathrm{~ms}$ (Wilcoxon signed rank test, $p<$ 0.001 ) and was significantly different from the median of the SR burst neurons (Mann-Whitney $U$ test, $p<0.001$ ), which was $11.5 \mathrm{~ms}$ after movement onset. The latter median was not significantly different from movement onset (Wilcoxon signed rank test, $p=0.06$ ). The two neurons which were responsive to both types of movements (distinguished by the gray bars in Fig. $9 B$ ) exhibited bursts which started very early with respect to movement onset positioning them outside the distribution of SR burst neurons and close to the left end of the one for the HMR burst 
neurons. The median burst durations of the two main classes of burst neurons were not significantly different from each other (SR burst neurons: $75.5(38,169) \mathrm{ms}$, HMR burst neurons: $63.5(22,180) \mathrm{ms}$, the quartiles are given in brackets; MannWhitney $U$ test: $p>0.05$ ).

\section{Responses of effector specific burst neurons during yoked eye and hand movements}

Although movement-related burst neurons typically did not respond to the movement of the nonpreferred effector this does not preclude the possibility that their response to the movement of the preferred effector is modulated by the need to move the nonpreferred effector in addition to the preferred one. To determine if this was the case, we analyzed the discharge of burst neurons to movements of the two effectors moving in isolation with responses to yoked eye and hand movements. Figure $6 B$ provides this comparison for the individual saccadic eye movement-related neuron, preferring downward saccades, whose direction tuning to single effector movements is shown in Figure $6 \mathrm{~A}$. During combined eye and hand movements this neuron did not show any change in the strength of its discharge or in its direction preference. The only change this neuron exhibited was a significant (Wilcoxon test, $p<0.05$ ) shortening of the burst duration in the case of yoked eye and hand movements compared with isolated saccades.

Figure $7 B$ presents the responses of the HMR burst neuron in the yoked condition, whose responses to single effector movements are depicted in Figure $7 A$. During combined eye and hand movements the discharge pattern of this neuron did not change when aligning it on hand movement onset compared with the response during isolated hand movements while a relatively pronounced postburst pause in firing could be observed. Unlike the previous example, in the case of the HMR burst neuron the timing of the bursts remained unaltered.

Figure 10 illustrates the dependence of the burst parameters on the two movement conditions (isolated vs yoked) for the two group of neurons firing either eye or hand movement-related bursts. In Figure $10 \mathrm{~A}$ the normalized burst strength $I_{\text {change }}$ for yoked eye and hand movements in the respective preferred direction is plotted as function of $I_{\text {change }}$ for isolated movements. Single effector burst neurons as well as the two neurons driven by both eye and hand movements are distinguished in this plot by their respective symbols. The two groups of single effector burst neurons (SR and HMR burst neurons) did not show a significant difference (paired Student's $t$ tests, $p>0.05$ corr., for SR and HMR neurons) of their burst strengths in the two conditions compared. Also the two neurons without any effector specificity (shown in gray) did not exhibit a significant change in their activity when comparing responses to yoked movements to those prompted by isolated eye or hand movements (paired Student's $t$ test, $p>0.05$ corr.). The conclusion that at most a few neurons showed an influence of the involvement of the second effector is also expressed by the fact that most of them fell on or close to the bisector of the plot. Figure $10 B-D$ analyzes the influence of involving a second effector in the action on various variables capturing the timing of the movement-related burst relative to movement onset (the latter indicated by the dashed horizontal and vertical lines). Similar to the preceding figure, they plot the variable at issue for the yoked movement as function of the same variable for the single effector case. The analysis of burst onset time (Fig. $10 \mathrm{~B}$ ) did not reveal significant differences between isolated and yoked movements for the effector selective SR and 


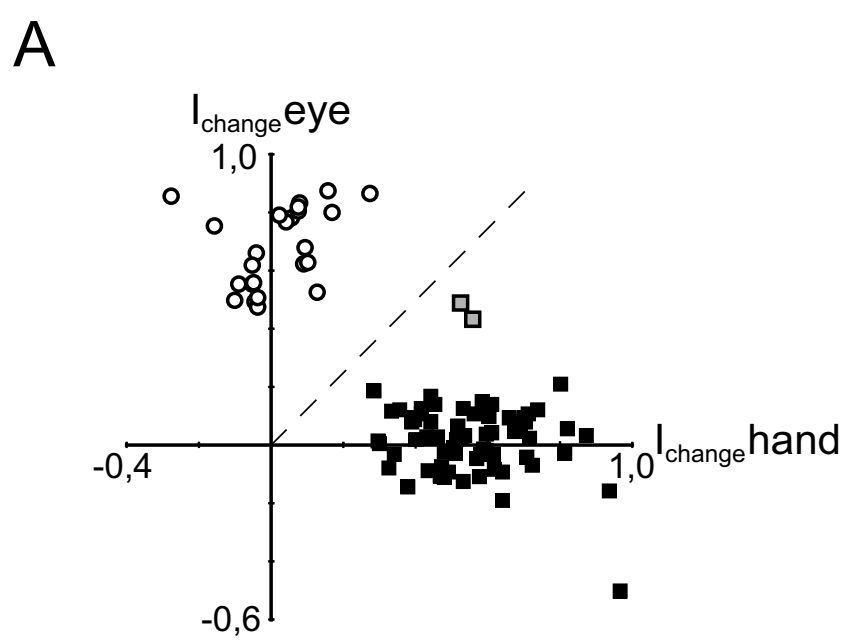

B

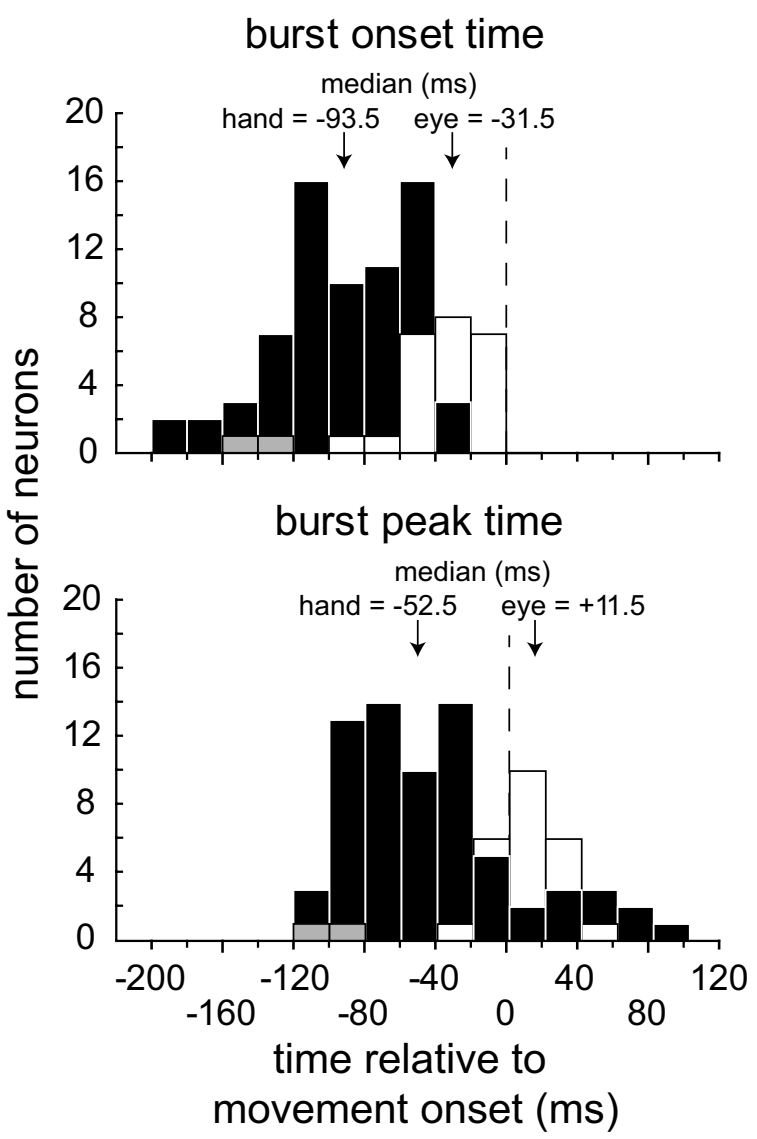

Figure 9. Population data of eye and/or hand movement-related units. $\boldsymbol{A}$, Plot of saccade-related responses ( $y$-axis) as a function of hand movement-related responses ( $x$-axis) based on the index $I_{\text {change }}$ capturing the change of spike rates relative to baseline levels during the burst intervals of saccadic eye movements ( $y$-axis) versus the index for hand movements ( $x$-axis) (see Materials and Methods). Negative values indicate a decrease of activity relative to baseline during the respective movement, whereas positive values reflect an increase of activity. Filled squares represent neurons that showed bursts for hand movements only, open circles those that responded to saccades only, and gray squares represent the two neurons responding to both types of movement. $\boldsymbol{B}$, Histograms (bin width $10 \mathrm{~ms}$ ) of burst onset (top) and burst peak time (bottom) relative to movement onset. Hand movement-related neurons are represented by black bars, saccade-related neurons by white bars, and the two neurons responding to both eye and hand movements by gray bars. The median latencies are given above the arrows.
HMR burst neurons (paired Student's $t$ tests, $p>0.05$ corr., for SR and HMR neurons). However, the two neurons that lacked effector specificity exhibited significantly earlier burst onset times for yoked movements compared with the two isolated movement conditions (paired Student's $t$ test, $p<0.05$ corr.).

For the timing of the peak of the bursts (Fig. 10C) as well as the overall duration of the bursts (Fig. 10D) both SR and HMR neurons showed a significant dependence on the movement condition characterized by earlier peaks and shorter durations for the yoked condition (paired Student's $t$ tests, $p<0.05$ corr., for SR and HMR neurons in both parameters). On the other hand, the two unspecific neurons did not differentiate between the two conditions (paired Student's $t$ test, $p>0.05$ corr., for both parameters).

\section{Discussion}

To clarify the role of the DPN in the planning and execution of goal-directed eye and hand movements, we recorded from PN neurons in two macaque monkeys, performing goal-directed eye movements, hand movements, or combinations of both, guided by spatial memory.

We could identify both hand and eye movement-related responses in two neighboring regions of the anterior part of the DPN. Both types of neurons could be found within a broad area along the medial to lateral axis involving regions of the DPN usually referred to as the dorsolateral, dorsal, and dorsomedial pontine nuclei. In general, HMR neurons were found somewhat deeper and more medial than EMR neurons.

Although our experiment focused on responses to memoryguided saccades, we also tested a substantial number of the EMR neurons in tasks requiring the execution of smooth pursuit eye movements. In agreement with a previous study (Dicke et al., 2004) the group of EMR neurons was heterogeneous. It included SR neurons, others preferring smooth pursuit as well as many neurons exhibiting sensitivity to both goal-directed eye movement types. The HMR neurons were similarly heterogeneous. While most of them started to discharge before the hand movement and usually stopped discharging as soon as the movement was over, others also responded to the presentation of the peripheral cue and/or showed an elevated level of activity in the memory period. Yet others encoded hand position in the frontoparallel plane rather than showing a response restricted to the hand movement. Although the movements were highly stereotypic, the fact that the movement-related bursts varied considerably in shape and latency between neurons speaks against a role in the encoding of movement kinematics or dynamics or the processing of proprioceptive signals. The heterogeneity seems more in line with a representation of higher level premotor information processing. Although some neurons in the sample exhibited clear visual responses elicited by the central fixation point or the peripheral spatial cue, we are confident that the bursts observed in conjunction with the movement were not elicited by inadvertent visual stimulation. First, the central cue had disappeared typically 190 to 160 ms before the onset of movement-related bursts, the latter usually well separated from an occasionally observable preceding target associated burst. Second, movement-related responses were in most cases directional. Hence, the disappearance of a central fixation dot serving as the start signal for the movement cannot account for the following directional response. Third, self-induced visual stimulation can be excluded. Assuming that dark adaptation might have allowed the monkeys to see their hand moving across the dark monitor, with a tiny amount of residual illumination, a hand movement induced visual response 
might be conceivable. However, this response should follow the movement, whereas movement-related bursts always led the movement. In sum, we can be confident that movement-related bursts are nonvisual.

Neuronal activity in the PN related to a specific aspect of forelimb movements has been reported previously (Matsunami, 1987). This author described neurons responding to simple extension-flexion movement at the wrist joint, either transiently or tonically. They were recorded in more medial parts of the PN than the region of HMR neurons delineated in our experiments. In view of the profound differences in the tasks in the two studies, we consider it very unlikely that the same pool of neurons were sampled. It seems more plausible that the two studies tapped functionally distinct types of HMR neurons in the DPN, contributing to different aspects of forearm movements and arguably dependent on different sources of cerebrocortical input. Cortical activity related to wrist movement has been described in a number of areas, among them primary motor cortex and premotor cortex (Kurata, 1993; Riehle and Requin, 1995; Kakei et al., 1999, 2001), projecting to more ventral parts of the PN (Brodal, 1978; Schmahmann et al., 2004a) not explored in the present study. A more likely source of HMR information in the DPN are axons originating from various posterior parietal areas, known to project to the dorsal parts of the PN, that contains neurons activated by hand reaches like area 5 (Ashe and Georgopoulos, 1994; Johnson et al., 1996; Scott et al., 1997; Hamel-Pâquet et al., 2006), area 7a (MacKay, 1992; Snyder et al., 1997; Merchant et al., 2004; BattagliaMayer et al., 2007), or the parietal reach area PRR (Batista and Andersen, 2001; Calton et al., 2002; Snyder et al., 2006).

Previous work on the architecture of the corticopontine projection of rodents strongly argues for parallel corticopontine channels, originating from distinct cerebrocortical areas, in turn contacting distinct patches of pontocerebellar neurons, which stay devoid of input from other parts of cortex (Schwarz and Thier, 1995; Schwarz and Möck, 2001) or functionally related subcortical structures (Schwarz et al., 2005). Preliminary evidence suggests that this organizational principle seems to apply to primates as well (Leichnetz et al., 1984; Huerta et al., 1986; Leichnetz and Gonzalo-Ruiz, 1996; Thier and Möck, 2006). Our physiological findings do not allow us to decide if HMR neurons in the DPN reflect input from individual parietal areas - as suggested by the anatomy-or a converging influence from many. In any case, the clear separation of two distinct visuomotor pools of neurons for hand and for eye movements in the DPN would clearly be more in line with the notion of parallel visuomotor channels.

These two populations of DPN neurons differed not only with
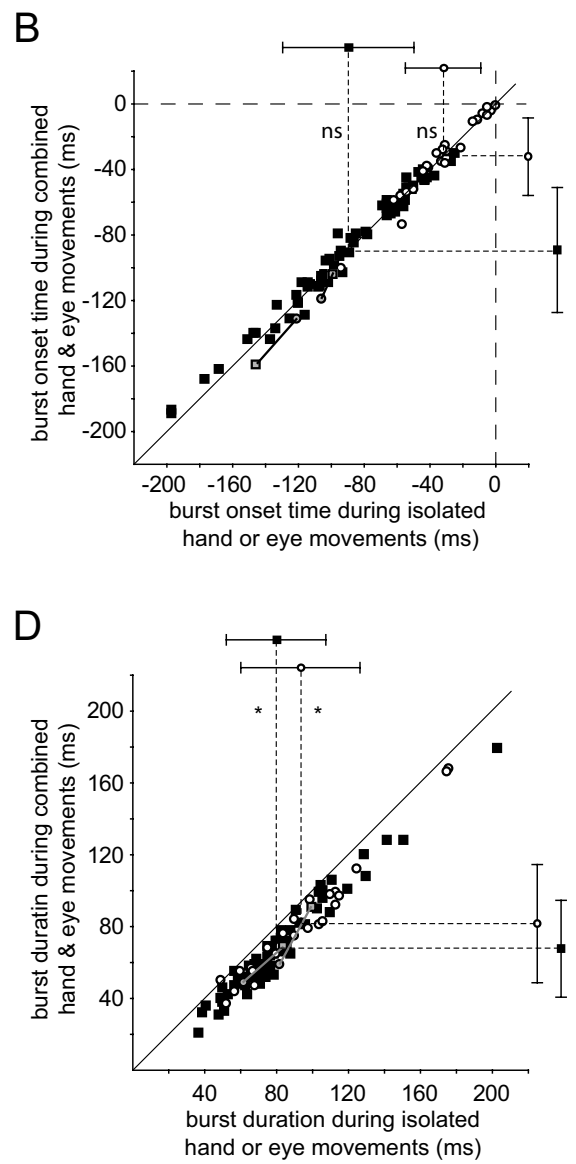

Figure 10. Comparison of strength $(\boldsymbol{A})$ and timing $(\boldsymbol{B})$ of movement-related bursts evoked by isolated and combined eye and hand movements. $\boldsymbol{A}$, Index $I_{\text {change }}$ of activity change relative to baseline for combined ( $y$-axis) versus isolated ( $x$-axis) movements. The data are aligned on the onset of the type of movement preferred by the respective neuron. Black squares depict HMR (w) two neurons activated by both types of movement are plotted in gray, aligned either on hand Bonferroni-corrected paired Student's $t$ tests. $\boldsymbol{B}$, Burst onset time for combined ( $y$-axis) versus isolated ( $x$-axis) movements, onset for combined versus isolated movements. Broken lines in $\boldsymbol{C}$ and $\boldsymbol{D}$ marked with asterisks denote significant differences, $p<$ 0.05. D, Burst duration for combined versus isolated movements. Notation is as in $\boldsymbol{C}$.

respect to the preferred action but also exhibited clear quantitative differences in the timing of the movement-related discharge. In both populations the onset of the movement-related burst preceded the onset of the movement. However, bursts of HMR neurons started on average $62 \mathrm{~ms}$ earlier than bursts of SR neurons, which led movement onset by on average $31 \mathrm{~ms}$, in accordance with previous observations (Dicke et al., 2004). The latency of burst onset times of HMR neurons in the DPN lies between the time range of possible cortical input structures like area 7 a where $61 \%$ of the HMR neurons precede the movement onset by $>200$ ms (Battaglia-Mayer et al., 2007) and motor cortex output that precedes movement initiation by 70-130 ms (Kakei et al., 1999). On the other hand, it leads those in lobules V and VI of the cerebellum where many neurons fire during movements (Liu et al., 2003), in full accordance with their role as intermediate stages in a pathway linking cerebral cortex and the cerebellum. The difference in burst onset time relative to movement onset between the SR and HMR neurons in the DPN is reminiscent of the differences in the time required to start an eye compared with a hand movement. Whereas it takes $2-11 \mathrm{~ms}$ from the onset of the 
discharge of an oculomotor neuron to the onset of the eye movement (Fuchs and Luschei, 1970), it may take up to $120 \mathrm{~ms}$ from the onset of the discharge of an HMR spinal motoneuron to the onset of any observable hand movement as the arm muscles need to be active several tens of milliseconds before movement start (Hasan and Karst, 1989; Flanders, 1991). In other words, the difference in burst onset times of the two classes of neurons may account for the different delays of signals to the two effectors.

Under natural conditions, successful reaches are prepared by first moving the eyes to the object of interest, thereby improving the visual information for the subsequent guidance of the hand (Biguer et al., 1982; Abrams et al., 1990; Bekkering et al., 1994). In such sequences saccades typically lead the hand by $50-100 \mathrm{~ms}$ (Snyder et al., 2002), reflecting tight central coordination of the movement plans involving the respective effectors. The need for effector coordination is not reflected in changes of the onset time or discharge strength of movement-related bursts of movementrelated DPN neurons. Rather, they exhibit very similar profiles, regardless of whether the movement of the preferred effector is joined by a movement of the respective other effector or not. The simplest explanation of the fact that discharge rates of effector specific movement-related neurons in the DPN ignore the need to move the second, nonpreferred effector would be a lack of coordination of the effectors. However, this is clearly not the case as the timing of the two effectors in the case of yoked movements was typically tightly coordinated. Hence, it seems more plausible that the neuronal adjustments required may involve changes of the temporal fine structure of discharge, possibly at the level of the cerebrocortical input, rather than changes of discharge rates at the level of the DPN.

In conclusion our findings support the notion of a distinct precerebellar, pontine visuomotor channel for hand reaches that is anatomically and functionally largely separated from the one serving eye movements.

\section{References}

Abrams RA, Meyer DE, Kornblum S (1990) Eye-hand coordination: oculomotor control in rapid aimed limb movements. J Exp Psychol Hum Percept Perform 16:248-267.

Ajne B (1968) A simple test for uniformity of a circular distribution. Biometrika 55:343-354.

Ashe J, Georgopoulos AP (1994) Movement parameters and neural activity in motor cortex and area 5. Cereb Cortex 4:590-600.

Batista AP, Andersen RA (2001) The parietal reach region codes the next planned movement in a sequential reach task. J Neurophysiol 85:539-544.

Battaglia-Mayer A, Ferraina S, Genovesio A, Marconi B, Squatrito S, Molinari M, Lacquaniti F, Caminiti R (2001) Eye-hand coordination during reaching. II. An analysis of the relationships between visuomanual signals in parietal cortex and parieto-frontal association projections. Cereb Cortex 11:528-544.

Battaglia-Mayer A, Mascaro M, Caminiti R (2007) Temporal evolution and strength of neural activity in parietal cortex during eye and hand movements. Cereb Cortex 17:1350-1363.

Bekkering H, Adam JJ, Kingma H, Huson A, Whiting HT (1994) Reaction time latencies of eye and hand movements in single- and dual-task conditions. Exp Brain Res 97:471-476.

Biguer B, Jeannerod M, Prablanc C (1982) The coordination of eye, head, and arm movements during reaching at a single visual target. Exp Brain Res 46:301-304.

Brodal P (1978) Principles of organization of the monkey corticopontine projection. Brain Res 148:214-218.

Buneo CA, Jarvis MR, Batista AP, Andersen RA (2002) Direct visuomotor transformations for reaching. Nature 416:632-636.

Calton JL, Dickinson AR, Snyder LH (2002) Non-spatial, motor-specific activation in posterior parietal cortex. Nat Neurosci 5:580-588.

Caminiti R, Ferraina S, Johnson PB (1996) The sources of visual informa- tion to the primate frontal lobe: a novel role for the superior parietal lobule. Cereb Cortex 6:319-328.

Dicke PW, Barash S, Ilg UJ, Thier P (2004) Single-neuron evidence for a contribution of the dorsal pontine nuclei to both types of target-directed eye movements, saccades and smooth-pursuit. Eur J Neurosci 19:609-624.

Flanders M (1991) Temporal patterns of muscle activation for arm movements in three-dimensional space. J Neurosci 11:2680-2693.

Fuchs AF, Luschei ES (1970) Firing patterns of abducens neurons of alert monkeys in relationship to horizontal eye movement. J Neurophysiol 33:382-392.

Gaymard B, Pierrot-Deseilligny C, Rivaud S, Velut S (1993) Smooth pursuit eye movement deficits after pontine nuclei lesions in humans. J Neurol Neurosurg Psychiatry 56:799-807.

Glickstein M, May JG 3rd, Mercier BE (1985) Corticopontine projection in the macaque: the distribution of labelled cortical cells after large injections of horseradish peroxidase in the pontine nuclei. J Comp Neurol 235:343-359.

Glickstein M, May J, Mercier B (1990) Visual corticopontine and tectopontine projections in the macaque. Arch Ital Biol 128:273-293.

Haaxma R, Kuypers HG (1975) Intrahemispheric cortical connexions and visual guidance of hand and finger movements in the rhusus monkey. Brain 98:239-260.

Hamel-Pâquet C, Sergio LE, Kalaska JF (2006) Parietal area 5 activity does not reflect the differential time-course of motor output kinetics during arm-reaching and isometric-force tasks. J Neurophysiol 95:3353-3370.

Hanes DP, Thompson KG, Schall JD (1995) Relationship of presaccadic activity in frontal eye field and supplementary eye field to saccade initiation in macaque: Poisson spike train analysis. Exp Brain Res 103:85-96.

Hasan Z, Karst GM (1989) Muscle activity for initiation of planar, two-joint arm movements in different directions. Exp Brain Res 76:651-655.

Hoover JE, Strick PL (1999) The organization of cerebellar and basal ganglia outputs to primary motor cortex as revealed by retrograde transneuronal transport of herpes simplex virus type 1. J Neurosci 19:1446-1463.

Hoshi E, Tremblay L, Féger J, Carras PL, Strick PL (2005) The cerebellum communicates with the basal ganglia. Nat Neurosci 8:1491-1493.

Huerta MF, Krubitzer LA, Kaas JH (1986) Frontal eye field as defined by intracortical microstimulation in squirrel monkeys, owl monkeys, and macaque monkeys: I. Subcortical connections. J Comp Neurol 253:415-439.

Johnson PB, Ferraina S, Bianchi L, Caminiti R (1996) Cortical networks for visual reaching: physiological and anatomical organization of frontal and parietal lobe arm regions. Cereb Cortex 6:102-119.

Judge SJ, Richmond BJ, Chu FC (1980) Implantation of magnetic search coils for measurement of eye position: an improved method. Vision Res 20:535-538.

Kakei S, Hoffman DS, Strick PL (1999) Muscle and movement representations in the primary motor cortex. Science 285:2136-2139.

Kakei S, Hoffman DS, Strick PL (2001) Direction of action is represented in the ventral premotor cortex. Nat Neurosci 4:1020-1025.

Kurata K (1993) Premotor cortex of monkeys: set- and movement-related activity reflecting amplitude and direction of wrist movements. J Neurophysiol 69:187-200.

Laule GE, Bloomsmith MA, Schapiro SJ (2003) The use of positive reinforcement training techniques to enhance the care, management, and welfare of primates in the laboratory. J Appl Anim Welf Sci 6:163-173.

Leichnetz GR, Gonzalo-Ruiz A (1996) Prearcuate cortex in the Cebus monkey has cortical and subcortical connections like the macaque frontal eye field and projects to fastigial-recipient oculomotor-related brainstem nuclei. Brain Res Bull 41:1-29.

Leichnetz GR, Smith DJ, Spencer RF (1984) Cortical projections to the paramedian tegmental and basilar pons in the monkey. J Comp Neurol 228:388-408.

Liu X, Robertson E, Miall RC (2003) Neuronal activity related to the visual representation of arm movements in the lateral cerebellar cortex. J Neurophysiol 89:1223-1237.

MacKay WA (1992) Properties of reach-related neuronal activity in cortical area 7A. J Neurophysiol 67:1335-1345.

Marconi B, Genovesio A, Battaglia-Mayer A, Ferraina S, Squatrito S, Molinari M, Lacquaniti F, Caminiti R (2001) Eye-hand coordination during reaching. I. Anatomical relationships between parietal and frontal cortex. Cereb Cortex 11:513-527. 
Matsunami K (1987) Neuronal activity in nuclei pontis and reticularis tegmenti pontis related to forelimb movements of the monkey. Neurosci Res 5:140-156.

Merchant H, Battaglia-Mayer A, Georgopoulos AP (2004) Neural responses during interception of real and apparent circularly moving stimuli in motor cortex and area 7a. Cereb Cortex 14:314-331.

Murata A, Gallese V, Luppino G, Kaseda M, Sakata H (2000) Selectivity for the shape, size, and orientation of objects for grasping in neurons of monkey parietal area AIP. J Neurophysiol 83:2580-2601.

Myers RE, Sperry RW, McCurdy NM (1962) Neural mechanisms in visual guidance of limb movement. Arch Neurol 7:195-202.

Riehle A, Requin J (1995) Neuronal correlates of the specification of movement direction and force in four cortical areas of the monkey. Behav Brain Res 70:1-13.

Rizzolatti G, Luppino G (2001) The cortical motor system. Neuron 31:889-901.

Sakata H, Taira M (1994) Parietal control of hand action. Curr Opin Neurobiol 4:847-856.

Schmahmann JD, Rosene DL, Pandya DN (2004a) Motor projections to the basis pontis in rhesus monkey. J Comp Neurol 478:248-268.

Schmahmann JD, Ko R, MacMore J (2004b) The human basis pontis: motor syndromes and topographic organization. Brain 127:1269-1291.

Schwarz C, Möck M (2001) Spatial arrangement of cerebro-pontine terminals. J Comp Neurol 435:418-432.

Schwarz C, Thier P (1995) Modular organization of the pontine nuclei: dendritic fields of identified pontine projection neurons in the rat respect the borders of cortical afferent fields. J Neurosci 15:3475-3489.

Schwarz C, Horowski A, Möck M, Thier P (2005) Organization of tectopon- tine terminals within the pontine nuclei of the rat and their spatial relationship to terminals from the visual and somatosensory cortex. J Comp Neurol 484:283-298.

Scott SH, Sergio LE, Kalaska JF (1997) Reaching movements with similar hand paths but different arm orientations. II. Activity of individual cells in dorsal premotor cortex and parietal area 5. J Neurophysiol 78:2413-2426.

Snyder LH, Batista AP, Andersen RA (1997) Coding of intention in the posterior parietal cortex. Nature 386:167-170.

Snyder LH, Calton JL, Dickinson AR, Lawrence BM (2002) Eye-hand coordination: saccades are faster when accompanied by a coordinated arm movement. J Neurophysiol 87:2279-2286.

Snyder LH, Dickinson AR, Calton JL (2006) Preparatory delay activity in the monkey parietal reach region predicts reach reaction times. J Neurosci 26:10091-10099.

Soechting JF, Flanders M (1989) Sensorimotor representations for pointing to targets in three-dimensional space. J Neurophysiol 62:582-594.

Suzuki DA, May JG, Keller EL, Yee RD (1990) Visual motion response properties of neurons in dorsolateral pontine nucleus of alert monkey. J Neurophysiol 63:37-59.

Thier P, Möck M (2006) The oculomotor role of the pontine nuclei and the nucleus reticularis tegmenti pontis. Prog Brain Res 151:293-320.

Thier P, Bachor A, Faiss J, Dichgans J, Koenig E (1991) Selective impairment of smooth-pursuit eye movements due to an ischemic lesion of the basal pons. Ann Neurol 29:443-448.

Wörgötter F, Daunicht WJ, Eckmiller R (1986) An on-line spike form discriminator for extracellular recordings based on an analog correlation technique. J Neurosci Methods 17:141-151. 